\title{
Experience-induced interocular plasticity of vision in infancy
}

\author{
Wayne W. Tschetter ${ }^{1}$, Robert M. Douglas ${ }^{2}$ and Glen T. Prusky ${ }^{1 *}$ \\ Department of Physiology and Biophysics, Burke Medical Research Institute, Weill Cornell Medical College, White Plains, NY, USA \\ 2 Department of Ophthalmology and Visual Science, University of British Columbia, Vancouver, BC, Canada
}

\author{
Edited by: \\ Raphael Pinaud, University of \\ Oklahoma Health Sciences Center, \\ USA \\ Reviewed by: \\ Elizabeth Quinlan, University of \\ Maryland College Park, USA \\ Benjamin D. Philpot, University of \\ North Carolina School of Medicine, \\ USA

\section{*Correspondence:} \\ Glen T. Prusky, Department of \\ Physiology and Biophysics, Burke \\ Medical Research Institute, Weill \\ Cornell Medical College, White Plains, \\ New York, NY 10605, USA. \\ e-mail: glp2004@med.cornell.edu
}

Animal model studies of amblyopia have generally concluded that enduring effects of monocular deprivation (MD) on visual behavior (i.e., loss of visual acuity) are limited to the deprived eye, and are restricted to juvenile life. We have previously reported, however, that lasting effects of MD on visual function can be elicited in adulthood by stimulating visuomotor experience through the non-deprived eye. To test whether stimulating experience would also induce interocular plasticity of vision in infancy, we assessed in rats from eye-opening on postnatal day $(P) 15$, the effect of pairing MD with the daily experience of measuring thresholds for optokinetic tracking (OKT). MD with visuomotor experience from P15 to P25 led to a 60\% enhancement of the spatial frequency threshold for OKT through the non-deprived eye during the deprivation, which was followed by loss-of-function ( $60 \%$ below normal) through both eyes when the deprived eye was opened. Reduced thresholds were maintained into adulthood with binocular OKT experience from P25 to P30. The ability to generate the plasticity and maintain lost function was dependent on visual cortex. Strictly limiting the period of deprivation to infancy by opening the deprived eye at P19 resulted in a comparable loss-of-function. Animals with reduced OKT responses also had significantly reduced visual acuity, measured independently in a discrimination task. Thus, experience-dependent cortical plasticity that can lead to amblyopia is present earlier in life than previously recognized.

Keywords: optomotor, optokinetic, plasticity, visual cortex, critical period, monocular deprivation, infancy

\section{INTRODUCTION}

Experience-dependent visual plasticity in mammals has long been associated with a "critical" period (CP) in juvenile life, in which monocular deprivation (MD) shifts cortical ocular dominance (OD) away from the deprived eye (Wiesel and Hubel, 1963; Hubel and Wiesel, 1970; Antonini and Stryker, 1993; Hensch, 2004). The clinical relevance of the plasticity is supported by evidence that the OD shift is accompanied by a loss of visual acuity (amblyopia) though the deprived eye, with little or no change through the non-deprived eye (Giffin and Mitchell, 1978; Prusky et al., 2000). More recent studies, however, have shown that effects of MD on visual cortex function are not limited to juvenile life. For example, cortical electrophysiology (Sawtell et al., 2003; Frenkel and Bear, 2004) and optical imaging studies (Hofer et al., 2006, 2009; Lehmann and Lowel, 2008; Sato and Stryker, 2008) in adult mice after the CP have reported MD-induced OD plasticity, which is expressed as an increase in the influence of the non-deprived eye. Analogous visual behavior experiments have reported that visual cortex-dependent function through the non-deprived eye is enhanced in adult mice during MD (Prusky et al., 2006), but have also revealed that stimulating visuomotor experience through the non-deprived eye during MD enables enhancement to endure after binocular vision is restored (Prusky et al., 2006; Lehmann and Lowel, 2008). Whereas these data show that different forms of OD plasticity exist in juvenile and adult life, they also suggest that the nature of visual experience through the non-deprived can dictate whether MD results in enduring behavioral change.
Whether experience through the non-deprived eye can facilitate effects of MD in infancy, before the $\mathrm{CP}$, is not known. Our previous behavioral studies in rodents have found that MD on its own in infancy does not affect visual acuity (measured in a visual discrimination task; Prusky et al., 2000) or visuomotor function (measured with optokinetic tracking, OKT; unpublished results). However, we were motivated by two lines of experimental evidence in rodents to investigate whether visuomotor experience would drive interocular plasticity of vision in infancy. First, we have reported (Prusky et al., 2008) that binocular visuomotor experience in rats from eye-opening can stimulate visual cortex-dependent enhancement of OKT responses and visual motion perception. This revealed that visual experience on its own could enable plasticity of spatial visual function before the effects of visual deprivation become evident in juvenile life. Second, mouse cortical imaging (Smith and Trachtenberg, 2007) and cellular activation (Tagawa et al., 2005) studies have reported atypical forms of binocular competition in the infant visual cortex, in which MD disrupts the refinement of retinotopy. Taken together, these results suggest that the quality of visual experience in infancy can affect the development of retinotopic fidelity necessary for spatial visual function.

In the present study, we thus investigated whether stimulating visuomotor experience through the non-deprived eye during MD in infancy would be capable of modifying spatial visual behavior. As previously, we utilized the quantification of spatial frequency (SF) and contrast thresholds for OKT in a virtual optokinetic system (Prusky et al., 2004; Douglas et al., 2005) as a means to expose rats to a transient, novel visuomotor experience that is tied to a 
rapid and adaptive motor response, and to measure visual system performance. In order to establish the clinical implications of our experiential manipulations we also measured the visual acuity of experimental and control animals in a grating discrimination task.

\section{MATERIALS AND METHODS}

Male and female Long-Evans rats from Charles River stock were reared at the Burke Medical Research Institute and housed on a 12-h light/dark cycle (lights on at 6 am). Pups were housed with their mother until postnatal day (P) 21, at which time they were divided into same-sex cohorts of two to four animals. We used the common definition of infant visual development as the period from eye-opening at $\mathrm{P} 15$ to weaning at $\mathrm{P} 21$, and juvenility from $\mathrm{P} 22$ until sexual maturity at $\sim \mathrm{P} 45$. The Cornell Institutional Animal Care and Use Committee (IACUC) authorized all procedures in this study.

Our method of quantifying OKT thresholds has been described previously (Prusky et al., 2004; Douglas et al., 2005). Briefly, vertical sine wave gratings (mean white $=266.46 \mathrm{~cd} / \mathrm{m}^{2}$; mean black $=0.29 \mathrm{~cd} / \mathrm{m}^{2}$ ) were projected on computer monitors as a virtual cylinder, with a circular platform positioned at the epicenter of the apparatus (OptoMotry@; CerebralMechanics). One at a time, animals were placed on the platform and an overhead video image was used to center the hub of the cylinder between the animal's eyes as it moved around, enabling the grating SF to be set at the animal's viewing position. When the cylinder was rotated $\left(12^{\circ} \mathrm{deg} / \mathrm{s}\right)$ and the animal tracked the stimulus with corresponding head and neck movements, it was judged that the animal could detect the grating. When measuring SF thresholds, a gray stimulus was projected whenever the animal groomed or walked about the platform. A trial was initiated when the animal paused, at which time the gray was replaced with a drifting grating, and tracking behavior was then assessed for as long as the animal remained still (usually a few seconds). The SF threshold was obtained using a method of limits procedure in which animals were exposed to SFs that incremented from $0.03 \mathrm{c} / \mathrm{deg}$, and decremented from $1.0 \mathrm{c} /$ deg, multiple times, until the highest SF that elicits tracking was identified. Since only temporal-to-nasal stimulus motion generates tracking (Douglas et al., 2005), thresholds through each eye were tested independently by altering the direction of cylinder rotation. Contrast thresholds were also measured in each testing session using similar procedures (data not shown). A complete set of thresholds through each eye was generated in 10-15 min. With the exception of the short period of OKT measurement, visual experience was not otherwise manipulated. Experimenters were "blind" to an animal's experimental condition and previously recorded thresholds, and adjunct observers were used regularly to validate threshold assessments.

We used a visual discrimination task (visual water task, VWT) to measure grating acuity, which has been described in detail previously (Prusky et al., 2000). Briefly, a trapezoidal-shaped tank was made into a Y-maze with a central divider at the wide end. A 30 " computer monitor spanned the end of the apparatus, which was used to project visual stimuli [a sine wave grating (mean white $=270.35 \mathrm{~cd} / \mathrm{m}^{2}$; mean black $=0.299 \mathrm{~cd} / \mathrm{m}^{2}$ ), or gray of the same mean luminance] into each arm through a glass wall. Water was added to the tank and an escape platform was submerged $2 \mathrm{~cm}$ beneath the surface, directly below the grating. Nothing was placed below the gray stimulus. Over a series of trials in which the R/L position of the grating and gray were varied pseudo-randomly, animals were trained to reliably discriminate between stimuli by swimming to the side displaying the grating, where they could escape from the water. Discrimination thresholds were obtained over trials by increasing the SF of the grating until animals failed to achieve 7/10 correct, and repeated at least three times. A cumulative normal curve was fit to performance as a function of SF, and the point on the curve that intersected with $70 \%$ correct was adopted as acuity. Visual stimuli were generated and experimental procedures were controlled with software (Acumen $\odot$; CerebralMechanics) processed on an Apple Mac Pro computer. Training in the VWT was initiated at P45 and acuity was obtained at or before P60. At the conclusion of acuity measurements, OKT thresholds were retested to confirm that they were unchanged by the VWT testing experience.

For MD, animals were placed under general anesthetic (inhaled isoflurane; induction at $2.5-4.5 \%$, maintenance at $1-2 \%$ evaporated in 1-1.5 L/min $\mathrm{O}_{2}$ ), a topical antibacterial ophthalmic agent (bacitracin-neomycin-polymyxin, BNP) was applied to the eyes, the middle 3-4 mm of the eyelid was trimmed and sewn ( 6.0 nylon) shut with pillow stitches placed below the eyelid margin, and the knots were sealed with cyanoacrylate glue. A topical anesthetic ( $0.5 \%$ proparacaine hydrochloride) was applied to the sutured eye and the animal was recovered on a warm pad. At the end of a deprivation period, animals were anesthetized and the stitches were removed. Eyes were checked regularly for clarity, and only animals with clear optics were included in the study.

For cortical ablations, animals were anesthetized with inhaled isoflurane (induction at 4-5\%, maintenance at $1.5-2.5 \%$ evaporated in $1-1.5 \mathrm{~L} / \mathrm{min}_{2}$ ) and placed in a stereotaxic frame. A topical antibacterial ophthalmic agent (BNP) was applied to the eyes, and the top of the head was shaved and then wiped with $10 \%$ povidoneiodine and 70\% ethanol. A midline incision was made in the scalp and the skin was resected to expose the skull. The boundaries of striate cortex were demarcated on the skull with reference to stereotaxic coordinates (Paxinos and Watson, 1998). A trephination was made over primary visual cortex (V1), the dura was resected and the cortex within the opening was aspirated down to white matter. Scalp incisions were closed with surgical staples and the animals were treated with an analgesic (buprenorphine, $0.05 \mathrm{mg} /$ $\mathrm{kg}$, subcutaneous). Animals were recovered on a warm pad, and once alert and mobile, they were returned to their home cages. Behavioral testing resumed the day after surgery. Upon the conclusion of behavioral testing, animals were anesthetized and perfused with buffered saline followed by buffered $4 \%$ paraformaldehyde. Brains were then removed, and the dorsal surface of each brain was photographed, traced, and superimposed to make the right panel illustrations in Figure 3.

\section{RESULTS}

In order to provide baseline assessments of visual function in the present study, we replicated our previous finding (Prusky et al., 2008) that daily OKT testing through both eyes from P15 (no visual deprivation) leads to enhancement of the OKT SF threshold through both eyes [( $\sim 60 \%$; Figure 1A, green trace $) 0.239 \mathrm{c} / \mathrm{deg}$ to $0.836 \pm 0.003 \mathrm{c} / \mathrm{deg}, n=4$ ], compared with equivalent testing experience from P25 [(Figure 1A, blue trace) $0.531 \pm 0.006 \mathrm{c} \mathrm{deg}$, 


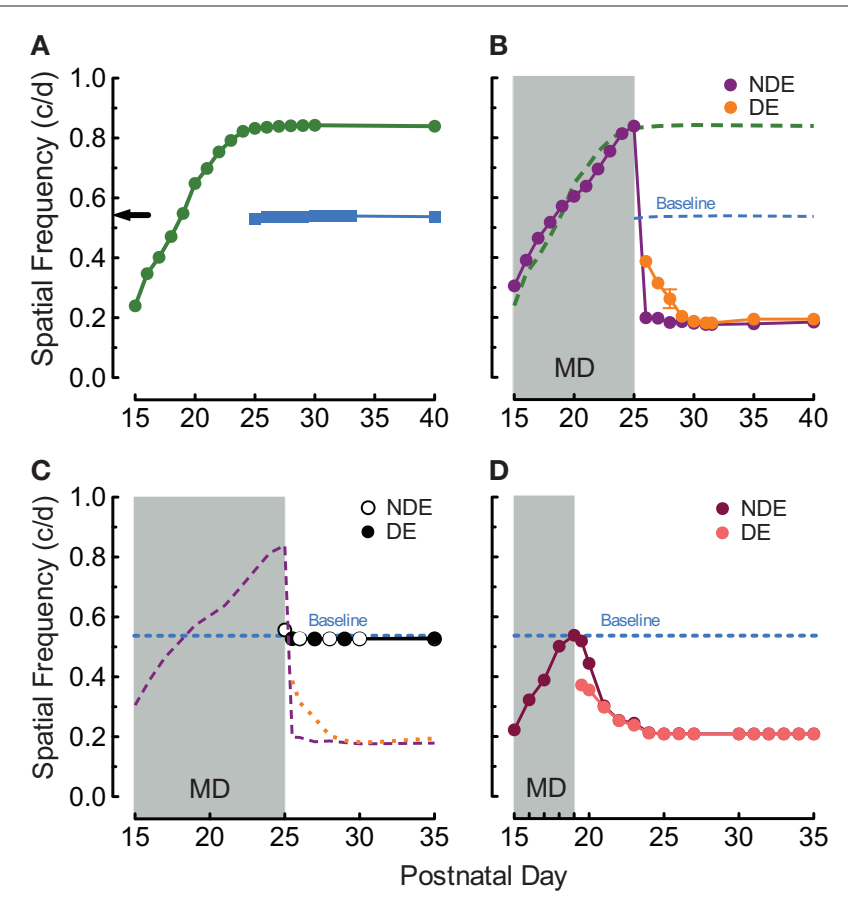

FIGURE 1 | Experience and MD-enabled visual plasticity from eyeopening. (A) The experience of measuring OKT through both eyes from P15 (green circles) leads to binocular enhancement of OKT SF thresholds ( 60\%, right eye responses plotted) that plateau at $\mathrm{P} 25$, relative to responses in naive adults (black arrow), or with testing from P25 (blue squares; Baseline; replication of Prusky et al., 2008). (B) P15-P25 MD with OKT experience led to enhancement through the non-deprived eye (NDE; purple trace) comparable to that with binocular experience (green dashed line from Figure 1A). Upon opening the deprived eye (DE; orange trace), however, thresholds through both eyes fell to $\sim 0.2 \mathrm{c} / \mathrm{deg}$ ( $\sim 60 \%$ reduction). (C) P15-P25 $\mathrm{MD}$ without OKT experience did not differ from baseline (orange, purple, and blue traces from Figure 1B). (D) P15-P19 MD with OKT experience was also sufficient to generate loss-of-function through both eyes. SEMs are plotted on this and other panels, but are often occluded by symbols. deprived eye on P25. This revealed that a slight enhancement was present during the MD (Figure 1C; first white dot; $0.558 \pm 0.005$ vs. $0.527 \pm 0.001 \mathrm{c} / \mathrm{deg}$ after $\mathrm{MD}, p<0.01, n=4)$, and after the MD (first black dot) thresholds were equal in both eyes, and comparable to subjects with binocular testing from P25 (0.527 \pm 0.001 vs. $0.538 \pm 0.001 \mathrm{c} / \mathrm{deg}, p=0.0279$, on P25, $n=4)$.

Since the rat CP has been reported to commence near P20 (Fagiolini et al., 1994; Guire et al., 1999), we wanted to eliminate the possibility that the deficits elicited with MD from P15 to P25 were due to the period of deprivation encroaching into the CP. We therefore instituted MD in animals from P15 to P19 $(n=5)$, an epoch of infancy before harmful effects of MD have been reported, in combination with OKT experience through the non-deprived eye. Figure 1D shows that when the deprived eye (salmon trace) was opened in these animals, thresholds through it, and the nondeprived eye (maroon trace), declined over the course of 5 days $(0.209 \pm 0.008 \mathrm{c} / \mathrm{deg})$ to the level of animals with MD up to P25 $(\sim 0.2 \mathrm{c} / \mathrm{deg})$.

We have previously reported that binocular enhancement of function in rats with OKT experience from P15 to P25 is consolidated into an enduring form with OKT experience from P25 to P30 (Prusky et al., 2008). We thus investigated whether MD from P15 to P30 accompanied by OKT experience, would enable enhancement through the non-deprived eye to endure after MD. To our surprise, we found (Figure 2A) that MD with OKT testing, even up to $\mathrm{P} 31$, was not able to maintain enhanced function; both eyes fell to near $0.2 \mathrm{c} / \mathrm{deg}(0.184 \pm 0.004 \mathrm{c} / \mathrm{deg}$ through the non-deprived and $0.191 \pm 0.018 \mathrm{c} / \mathrm{deg}$ through the deprived eye on P35, $n=4$ ) after the deprivation period. In order to test whether P25-P30 binocular testing experience was necessary to consolidate loss-of-function after $\mathrm{MD}$, we suspended testing from P25 to P30, which led to almost full recovery of OKT thresholds by P30 $(0.487 \pm 0.011$ vs. $0.535 \pm 0.003 \mathrm{c} /$ deg, $p<0.001$, Figure 2B, open and closed circles, $n=3$ ).

Enhancement generated and consolidated with binocular OKT experience in rats from P15 to P30 depends on the function of visual cortex (Prusky et al., 2008). In order to determine whether

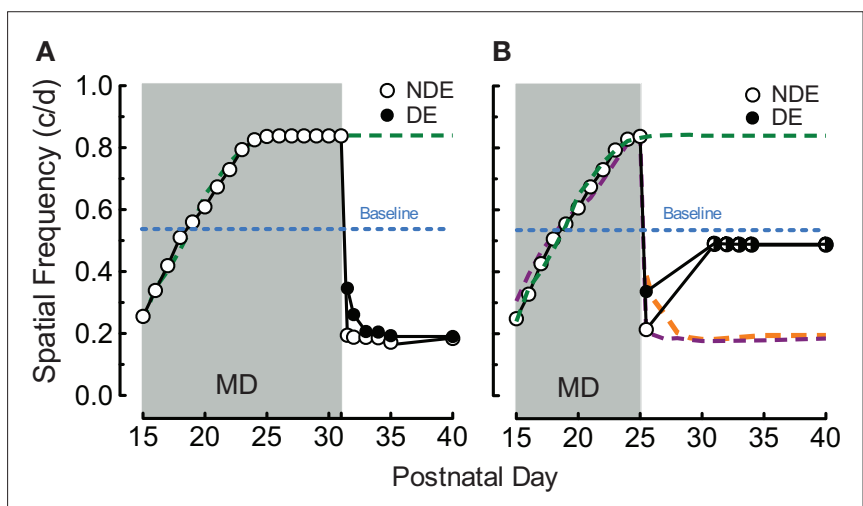

FIGURE 2 | Juvenile experience consolidates loss-of-function. (A) OKT experience after P25 was not sufficient to consolidate enhancement through the NDE (as binocular testing does; i.e., Prusky et al., 2008) and prevent loss-of-function (green and blue traces from Figure 1A). (B) Binocular OKT experience after P15-P25 MD was necessary to maintain loss-of-function (as in Figure 1B), since thresholds returned to near baseline when OKT testing was suspended from P25 to P30. 
the changes generated by pairing MD with OKT experience from P15 (i.e., Figures 1B,D) are also dependent upon the visual cortex, we made bilateral V1 lesions in 14-day-old rats, and instituted MD and OKT testing at P15 $(n=4)$. Cortex removal blocked enhancement of function through the non-deprived eye $(0.543$ vs. $0.839 \mathrm{c} / \mathrm{deg}$ with cortex and testing on P25; Figure 3A, open circles) during the $\mathrm{MD}$, and also mitigated the loss-of-function through both eyes after the MD (falling only to $0.508 \mathrm{vs} .0 .181 \mathrm{c} /$ deg, Figure 3A). We also tested whether loss-of-function after MD was dependent on visual cortex, by ablating V1 bilaterally on P30 in animals with combined MD and OKT experience from P15 to $\mathrm{P} 25(n=3)$. To our surprise, this manipulation resulted in an asymmetric response; thresholds through the previously deprived eye improved to baseline values soon after the lesion (Figure 3B, closed circles, $0.523 \mathrm{c} / \mathrm{deg}$ by P35), with function through the previously non-deprived eye remaining nearly unchanged. We detected no change in the temporal-to-nasal bias in OKT through an eye (Douglas et al., 2005) as the result of our experimental manipulations.

In order to determine whether animals with reduced OKT thresholds were amblyopic (Figures 4 A,B is the timeline and testing schedule for groups in A), we compared grating acuity in naïve adult rats (Naïve), rats with MD alone from P15 to P19 (MD- 15-19), rats with MD from P15 to P19 with OKT experience (MD+15-19), and rats with MD from P15 to P25 with OKT experience $(\mathrm{MD}+15-25)$. Our analysis revealed a significant effect

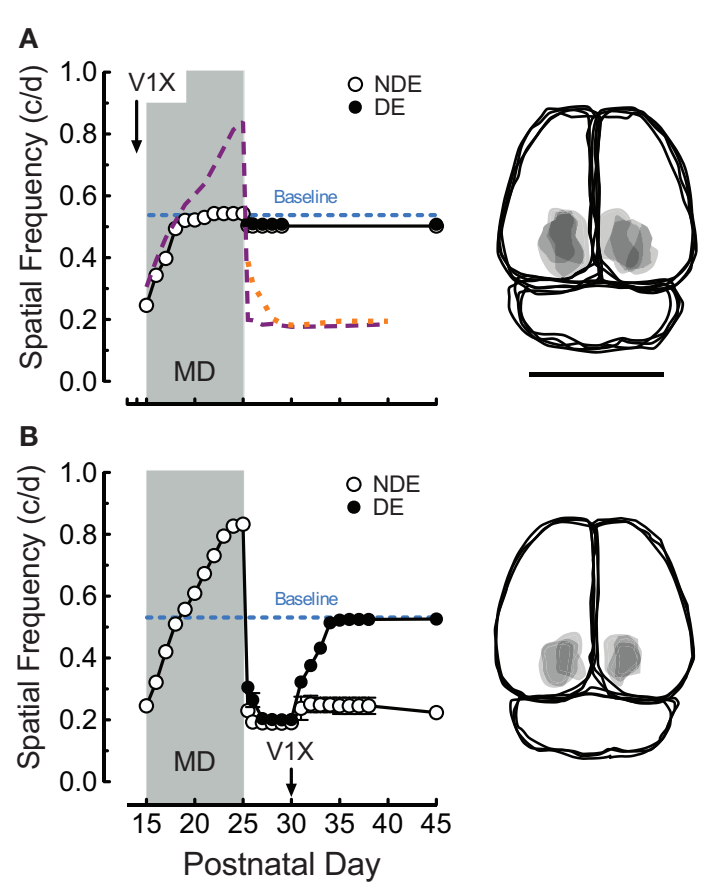

FIGURE 3 | Monocular deprivation and experience-enabled changes from P15 depend on visual cortex. (A) Bilateral V1 ablation on P14 prevented both enhancement, and loss-of-function generated by combined MD and OKT testing from P15 (orange, purple, and blue traces from Figure 1B). Right panels are overlapping traces of lesions in groups. Bar $=1 \mathrm{~cm}$. (B) Bilateral V1 ablation at P30 enabled thresholds through the previously deprived eye, but not the non-deprived eye, to recover. of group (one-way ANOVA, $F_{(3,28)}=15.61, P<0.0001$ ). Post hoc tests showed that MD- 15-19 animals did not have significantly lower thresholds than Naïve animals (MD- 15-19, $1.05 \pm 0.043 \mathrm{c}$ / $\operatorname{deg}(n=9)$ vs. Naïve, $1.16 \pm 0.030 \mathrm{c} / \operatorname{deg}(n=6), P>0.05$; Tukey's multiple comparison test). This showed that MD in infancy from P15 to P19 did not reduce visual acuity, supporting the notion that experiential manipulations up to P19 do not encroach on the CP. Both MD+ 15-19 and MD+ 15-25 groups, however, had significantly reduced acuity, compared with Naïve and MD$15-19$ groups $[\mathrm{MD}+15-19,0.873 \pm 0.041 \mathrm{c} / \mathrm{deg}(n=9)$ vs. Naïve, $1.16 \pm 0.030 \mathrm{c} / \mathrm{deg}(n=6), p<0.05 ; \mathrm{MD}+15-25,0.740 \pm 0.057 \mathrm{c} /$ $\operatorname{deg}(n=8)$ vs. Naïve, $1.16 \pm 0.030 \mathrm{c} / \operatorname{deg}(n=6), P<0.05 ; \mathrm{MD}+$ $15-19,0.873 \pm 0.041 \mathrm{c} / \mathrm{deg}(n=9)$ vs. MD- $15-19,1.05 \pm 0.043 \mathrm{c} /$ $\operatorname{deg}(n=9), p<0.05 ; \mathrm{MD}+15-25,0.740 \pm 0.057 \mathrm{c} / \mathrm{deg}(n=8) \mathrm{vs}$. MD- 15-19, $1.05 \pm 0.043 \mathrm{c} / \mathrm{deg}(n=9), P<0.05]$. Taken together, these data showed that visuomotor experience stimulated maladaptive effects of MD in infancy.

\section{DISCUSSION}

We have previously characterized an era of infant plasticity in rats in which visuomotor experience can enable visual function (Prusky et al., 2008). This work, in combination with evidence of binocular plasticity in infancy (Tagawa et al., 2005; Smith and Trachtenberg, 2007), motivated us to investigate whether effects of MD would be present in infancy if visuomotor experience was actively stimulated through the non-deprived eye. Indeed, we found that the nature of visual experience can stimulate interocular plasticity of vision in infancy: the experience of OKT threshold testing enabled a gain-of-function through the nondeprived eye during MD, followed by a loss-of-function through both eyes when binocular vision was restored. Therefore, two manifestations of experience-dependent plasticity of vision appear to be in effect prior to the CP: one in which visual experience enables visual function, and another in which visual experience drives interocular interactions. The nature of visual experience in infancy thus appears to dictate whether the visual cortex plays a role in enhancing, or disabling, visual function. As such, the results carry clinical implications for the prevention and treatment of developmental visual disorders.

Previous visual plasticity studies in rodents have provided evidence that two distinct processes govern when and how experience shapes the development of cortical visual function. The first, based on the results of numerous visual deprivation studies (for reviews see Cynader, 1982; Hensch, 2005), is that a transient period of binocular competition is present in juvenile life; "interocular" plasticity. The second, based on the results of stimulating visuomotor experience with both eyes open rather than inducing visual deprivation, is that a distinct period of plasticity is present in advance of the $\mathrm{CP}$ in infancy, which is characterized by the ability of visuomotor experience to enable a gain of visual function (i.e., Prusky et al., 2008); "enabling" plasticity. We propose that during the juvenile $\mathrm{CP}$ when an interocular form of plasticity dominates, MD on its own is sufficient to de-correlate activity in the brain between the eyes, which leads to loss-of-function through the deprived eye. However, in infancy when an enabling form of plasticity prevails, the stimulation of visuomotor experience on its own leads to a gain of visual function, regardless of whether experience was directed through one or both eyes (Prusky et al., 2008). MD in infancy in the absence of stimulated experience would not lead to a change 


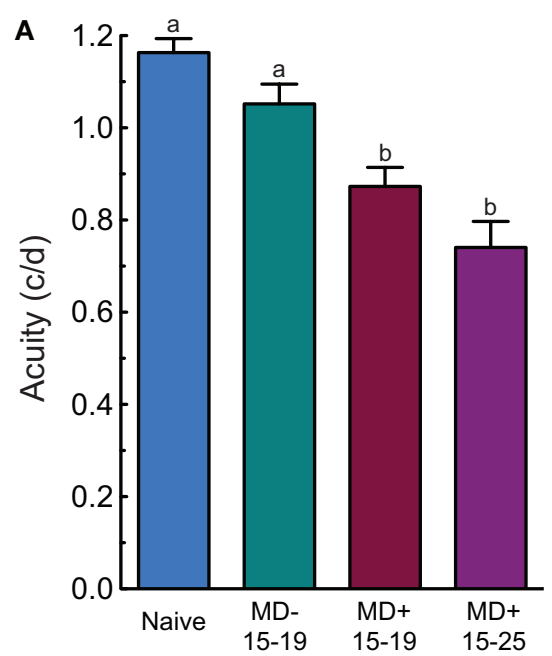

B

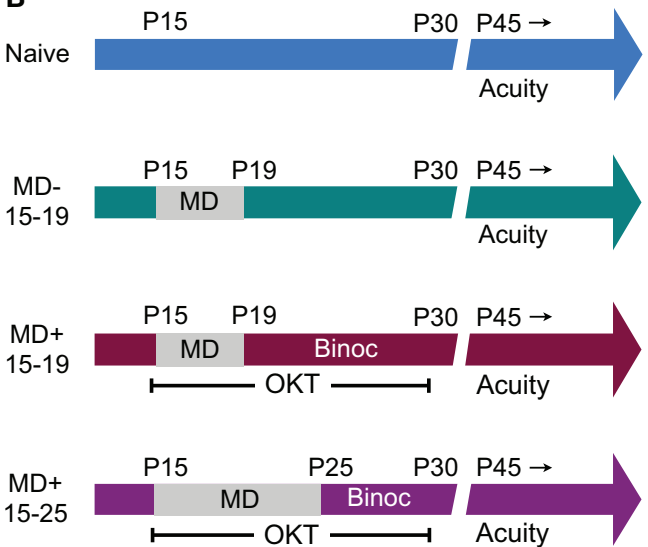

FIGURE 4 | Experience-enabled loss of OKT is accompanied by amblyopia. (A) MD on its own in infancy (MD-15-19) did not lead to reduced acuity in adulthood, relative to naive controls (Naive). However, adult acuity was lower in groups with MD and OKT experience up to P19 (MD+15-19; i.e., Figure 1D) or P25 (MD+15-25; i.e., Figure 1B). a vs. b indicates statistical significance, $p<0.05$. (B) Experiential timeline and visual testing schedule for groups in (A). OKT testing was monocular during the MD and Binocular (Binoc) after, in MD+ groups.

of function, since it would not sufficiently de-correlate activity between the eyes to enable interocular plasticity to prevail over enabling plasticity. Visuomotor stimulation through the non-deprived eye, however, would provide sufficient activation to de-correlate activity, thereby strengthening the normally diminutive interocular form of plasticity.

How visual experience affects the development of cortical circuits in infancy is not known. Work by Tagawa et al. (2005) and Smith and Trachtenberg (2007), has shown that the distinction between infant and juvenile forms of rodent visual plasticity is not absolute, by providing evidence that binocular competition is present in infancy, but with different characteristics than that during the CP; It is detectable not by a typical shift in OD or the loss of vision through a deprived eye, but by activity-dependent changes in retinotopy induced by MD. The present study supports the idea that experience-dependent binocular interactions are present in infancy, but expands on the evidence by showing that when visuomotor experience is combined with MD in infancy, changes in function are induced that ultimately result in loss of spatial visual function. It thus appears that the effects on cortical retinotopy of infant MD alone are not sufficient to effect a change in visual behavior. Since ipsilateral cortical maps appear to be most sensitive to binocular plasticity in infancy (Faguet et al., 2009), our visuomotor experience may produce a reduction of retinotopic fidelity sufficient to produce visual dysfunction.

We have previously reported that circuits in visual cortex are experimentally "necessary" and "sufficient" to support experienceinduced gain of visual function in infant rats when both eyes are open (i.e., Figure 1A; Prusky et al., 2008). Since the interocular plasticity we characterized in the present study was defined by comparable enhancement of function through the non-deprived eye during $\mathrm{MD}$, we were not surprised that ablations of visual cortex blocked enhancement (Figure 3A). The gain-of-function likely depends on the modulation of accessory optic system (AOS; retinal image stabilizing) circuits through descending cortical projections that are capable of mediating higher SFs (Grasse et al., 1984; Simpson, 1984; Grasse and Cynader, 1988; Natal and Britto, 1988; Distler and Hoffmann, 1992; Schmidt et al., 1993; Shintani et al., 1999; Masson et al., 2001; Hoffmann et al., 2002). We were, however, surprised that $\mathrm{V} 1$ lesions in animals with reduced function facilitated recovery of function through the previously deprived eye (Figure 3B). This indicates that the effects of visuomotor experience are bi-directional in nature; in the absence of unilateral visual deprivation, experience can shape cortical circuits to respond to higher SF stimuli, but when it accompanies MD it can also instruct cortical circuits to suppress visual function. We were also surprised that the bilateral removal of primary visual cortex did not affect function through the eyes equally. That the previously non-deprived eye does not recover function when the deprived eye does, shows that combining MD with visuomotor experience in infancy has an asymmetric influence on visual cortical function that is not evident in visual behavior when both eyes are open. In addition, it suggests that circuits outside of visual cortex differentially regulate visual behavior through the previously deprived and non-deprived eyes. This asymmetry may also be evident in the rate of functional decline after MD, with the previously non-deprived eye maximally losing function immediately, and the previously deprived eye declining to the same level only after 4 days of binocular experience (Figure 1B). Thus, visual behavior may not always be linearly related to the experiential history of an eye, but can be dictated by system-level interactions in the brain, which in the case of combined MD and visuomotor experience in infancy, serve to equalize behavioral function through the eyes.

Linking specific circuits and synaptic changes in the visual cortex with visual function has historically proven to be a challenge, and dissecting the cellular substrates of the experience and cortex enabled changes in visual function we have reported is well beyond the scope of this study. However, the obvious learning like properties of the plasticity combined with evidence that experience-dependent enhancement of visual function is dependent on cortical activity (Prusky et al., 2006, 2008) and NMDA receptor function (Sawtell et al., 2003), suggests that our experienceenabled loss-of-function through the deprived eye may be the 
result of synaptic changes in binocular visual cortex predicted by the BCM learning rule (Bienenstock et al., 1982; Kind, 1999). In this model, the temporal patterning associated with normal binocular activity is capable of maintaining a relatively high threshold for the synaptic modification of each eye's cortical input when interocular plasticity is in effect. This competition would enable the development of cortical cells with shared OD, and shape the receptive fields (i.e., orientation and SF selectivity) to support competent spatial visual function. The same competitive interaction would also result in the active removal of receptive fields with low precision. Thus, if both eyes are open in infancy when enabling plasticity is pronounced (i.e., Figure 1A), balanced visuomotor experience would serve to augment the threshold for the synaptic modification of each eye's influence in cortex, favoring the development of higher precision receptive fields and the enhancement of spatial visual function (i.e., Figure 1A). If, as we suggest, visuomotor experience during MD in infancy augments a normally diminutive form of interocular plasticity, the patterned activity would serve to potentiate non-deprived eye circuits, but the lower quality experience through the deprived eye would not be sufficient to maintain the modification threshold at a high value, resulting in the active depression of the deprived

\section{REFERENCES}

Antonini, A., and Stryker, M. P. (1993). Rapid remodeling of axonal arbors in the visual cortex. Science 260, 1819-1821.

Bienenstock, E. L., Cooper, L. N., and Munro, P. W. (1982). Theory for the development of neuron selectivity: orientation specificity and binocular interaction in the cortex. J. Neurosci. 2, 232-248.

Cynader, M. S. (1982). Competitive neuronal interactions underlying amblyopia. Hum. Neurobiol. 1, 35-39.

Distler, C., and Hoffmann, K. P. (1992). Early development of the subcortical and cortical pathway involved in optokinetic nystagmus: the cat as a model for man? Behav. Brain Res. 49, 69-75.

Douglas, R. M., Alam, N. M., Silver, B. D., McGill, T. J., Tschetter, W. W., and Prusky, G. T. (2005). Independent visual threshold measurements in the two eyes of freely moving rats and mice using a virtual-reality optokinetic system. Vis. Neurosci. 22, 677-684.

Fagiolini, M., Pizzorusso, T., Berardi, N., Domenici, L., and Maffei, L. (1994). Functional postnatal development of the rat primary visual cortex and the role of visual experience: dark rearing and monocular deprivation. Vision Res. 34, 709-720.

Faguet, J., Maranhao, B., Smith, S. L., and Trachtenberg, J. T. (2009). Ipsilateral eye cortical maps are uniquely sensitive to binocular plasticity. J. Neurophysiol. 101, 855-861.

Frenkel, M.Y., and Bear, M.F. (2004). How monocular deprivation shifts ocular dominance in visual cortex of young mice. Neuron 44, 917-923.

Giffin, F., and Mitchell, D. E. (1978). The rate of recovery of vision after early monocular deprivation in kittens. J. Physiol. (Lond.) 274, 511-537.

Grasse, K. L., and Cynader, M. S. (1988). The effect of visual cortex lesions on vertical optokinetic nystagmus in the cat. Brain Res. 455, 385-389.

Grasse, K. L., Cynader, M. S., and Douglas, R. M. (1984). Alterations in response properties in the lateral and dorsal terminal nuclei of the cat accessory optic system following visual cortex lesions. Exp. Brain Res. 55, 69-80.

Guire, E. S., Lickey, M. E., and Gordon, B. (1999). Critical period for the monocular deprivation effect in rats: assessment with sweep visually evoked potentials. J. Neurophysiol. 81, 121-128.

Hensch, T. K. (2004). Critical period regulation. Annu. Rev. Neurosci. 27, 549-579.

Hensch, T. K. (2005). Critical period mechanisms in developing visual cortex. Curr. Top. Dev. Biol. 69, 215-237.

Hofer, S. B., Mrsic-Flogel, T. D., Bonhoeffer, T., and Hubener, M. (2006). Prior experience enhances plasticity in the adult visual cortex. Nat. Neurosci. 1, 127-132.

Hofer, S. B., Mrsic-Flogel, T. D., Bonhoeffer, T., and Hubener, M. (2009). Experience leaves a lasting structural trace in cortical circuits. Nature 7227, 313-317.

Hoffmann, K. P., Bremmer, F., Thiele, A., and Distler, C. (2002). Directional

eye's synapses and the selection of lower quality receptive fields. Thus, when the deprived eye is opened, it would have reduced visual function (i.e., Figure 1B). An explanation of why the nondeprived eye loses function after it has been enhanced during MD does not appear to fit within the framework of the BCM theory. Instead, its reduced function is likely the result of maladaptive interactions between the eyes in circuits outside of binocular visual cortex that result in visual dysfunction only after balanced drive through each eye is restored after MD.

Finally, our findings likely have clinical implications, since they support the possibility that developmental visual disorders, such as amblyopia, could result from atypical visual experience earlier in life than has been appreciated previously. In addition, much consideration has been given to the diagnosis of imbalances in ocular experience early in life, and monocular patching is often used to treat these imbalances (Mitchell, 2008; Schmucker et al., 2010). However, little attention has been paid to the nature of visual experience through the non-patched eye. Our results indicate that caution should be exercised in patching treatments, since the relationship between age, visual deprivation, and visual experience, is complicated, and concomitant visual experience may contribute to dysfunction.

asymmetry of neurons in cortical areas MT and MST projecting to the NOTDTN in macaques. J. Neurophysiol. 87, 2113-2123.

Hubel, D. H., and Wiesel, T. N. (1970). The period of susceptibility to the physiological effects of unilateral eye closure in kittens. J. Physiol. (Lond.) 206, 419-436.

Kind, P. C. (1999). Cortical plasticity: is it time for a change? Curr. Biol. 9 , R640-R643.

Lehmann, K., and Lowel, S. (2008). Agedependent ocular dominance plasticity in adult mice. PLoS ONE 3, e3120. doi: 10.1371/journal.pone.0003120

Masson, G. S., Busettini, C., Yang, D. S. and Miles, F. A. (2001). Short-latency ocular following in humans: sensitivity to binocular disparity. Vision Res. 41, 3371-3387.

Mitchell, D. E. (2008). A special role for binocular visual input during development and as a component of occlusion therapy for treatment of amblyopia. Restor. Neurol. Neurosci. 26, 425-434.

Natal, C. L., and Britto, L. R. (1988). The rat accessory optic system: effects of cortical lesions on the directional selectivity of units within the medial terminal nucleus. Neurosci. Lett. 91, 154-159.

Paxinos, G., and Watson, C. (1998). The Rat Brain in Stereotaxic Coordinates, 4th Edn. San Diego: Academic Press.

Prusky, G. T., Alam, N. M., Beekman, S., and Douglas, R. M. (2004). Rapid quantification of adult and developing mouse spatial vision using a virtual optomotor system. Invest. Ophthalmol. Vis. Sci. 45, 4611-4616.

Prusky, G. T., Alam, N. M., and Douglas, R. M. (2006). Enhancement of vision by monocular deprivation in adult mice. J. Neurosci. 26, 11554-11561.

Prusky, G. T., Silver, B. D., Tschetter, W. W., Alam, N. M., and Douglas, R. M. (2008). Experience-dependent plasticity from eye-opening enables lasting, visual cortex-dependent enhancement of motion vision. J. Neurosci. 28, 9817-9827.

Prusky, G. T., West, P. W., and Douglas, R. M. (2000). Experience-dependent plasticity of visual acuity in rats. Eur. J. Neurosci. 12, 3781-3786.

Sato, M., and Stryker, M. P. (2008). Distinctive features of adult ocular dominance plasticity. J. Neurosci. 28 , 10278-10286.

Sawtell, N. B., Frenkel, M. Y., Philpot, B. D., Nakazawa, K., Tonegawa, S., and Bear, M. F. (2003). NMDA receptordependent ocular dominance plasticity in adult visual cortex. Neuron 38, 977-985.

Schmidt, M., Zhang, H.Y., and Hoffmann, K. P. (1993). OKN-related neurons in the rat nucleus of the optic tract and dorsal terminal nucleus of the accessory optic system receive a direct cortica input. J. Comp. Neurol. 330, 147-157.

Schmucker, C., Kleijnen, J., Grosselfinger, R., Riemsma, R., Antes, G., Lange, S., and Lagreze, W. (2010). Effectiveness of early in comparison to late(r) treatment in children with amblyopia or its risk factors: a systematic review. Ophthalmic. Epidemiol. 17, 7-17. 
Shintani, T., Hoshino, K., Meguro, R., Kaiya, T., and Norita, M. (1999). A light and electron microscopic analysis of the convergent retinal and visual cortical projections to the nucleus of the optic tract (NOT) in the pigmented rat. Neurobiology (Bp) 7, 445-460.

Simpson, J. I. (1984). The accessory optic system. Annu. Rev. Neurosci. 7, 13-41.

Smith, S. L., and Trachtenberg, J. T. (2007).

Experience-dependent binocular com- petition in the visual cortex begins at eye-opening. Nat. Neurosci. 10,370-375.

Tagawa, Y., Kanold, P. O., Majdan, M., and Shatz, C. J. (2005). Multiple periods of functional ocular dominance plasticity in mouse visual cortex. Nat. Neurosci. 8, 380-388.

Wiesel, T. N., and Hubel, D. H. (1963). Single-cell responses in striate cortex of kittens deprived of vision in one eye. J. Neurophysiol. 26, 1003-1017.
Conflict of Interest Statement: Glen T. Prusky and Robert M. Douglas are principals in a company (CerebralMechanics, Inc.) that markets equipment used in this study.

Received: 30 March 2011; accepted: 01 June 2011; published online: 15 June 2011.

Citation: Tschetter WW, Douglas RM and Prusky GT (2011) Experience-induced interocular plasticity of vision in infancy.
Front. Syst. Neurosci. 5:44. doi: 10.3389/ fnsys.2011.00044

Copyright (ㅇ 2011 Tschetter, Douglas and Prusky. This is an open-access article subject to a non-exclusive license between the authors and Frontiers Media SA, which permits use, distribution and reproduction in other forums, provided the original authors and source are credited and other Frontiers conditions are complied with. 